\title{
Moduli of Elastomeric Networks Prepared by Tetrafunctionally Endlinking Vinyl-Terminated Poly(dimethylsiloxane) Chains at Low Temperature
}

\author{
Mohammed A. Sharaf, ${ }^{*}$ James E. Mark, and A. S. Alshamsi**,† \\ Department of Chemistry and the Polymer Research Center, The University of Cincinnati, \\ Cincinnati, $\mathrm{OH} 45221-0172$, U.S.A. \\ * Department of Chemistry, Cairo University at Beni-Suef, Beni-Suef, Egypt \\ ** Department of Applied and Engineering Sciences, Vanderbilt University, \\ Nashville, TN 37235, U.S.A.
}

(Received May 29, 1995)

\begin{abstract}
Poly(dimethylsiloxane) (PDMS) networks have been prepared by tetrafunctionally endlinking vinyl-terminated chains, in a hydrosilylation cure at low temperatures. The results obtained demonstrated the absence of important side reactions, and the resulting networks were studied with regard to their stress-strain isotherms in elongation. Values of the moduli in the large deformation (phantom) limit and small deformation (affine) limit fell within the bounds predicted by the constrained-junction theory. The results do not suggest the presence of significant contributions from trapped entanglements. In this analysis, factors affecting the determination of the network structural parameters have been emphasized, because of serious experimental difficulties in making perfect networks. It appears that, in the absence thus far of carefully controlled and well-defined stoichiometry in the reacting systems, the procedure best suited for testing the various theories of rubberlike elasticity consists of plotting the sum of the Mooney-Rivlin constants $2 C_{1}+2 C_{2}$ (representing the low-deformation) modulus against $2 C_{1}$ (representing the high-deformation phantom modulus).
\end{abstract}

KEY WORDS Model Networks / Poly(dimethylsiloxane) / Endlinking / Hydrosilylation Cure Mechanical Properties / Stress-Strain Isotherms / Elongation Moduli / Rubber Elasticity Theories /

In recent years, a considerable number of studies have been carried out on model networks of poly(dimethylsiloxane) (PDMS). A central goal of these studies was to test the applicability of the various theories of rubberlike elasticity. ${ }^{1-24}$ By employing end-linking reactions, the number of strands between junctions and the functionalities of the junctions can be calculated. These techniques usually involve the reaction between bifunctional polymer chains and multifunctional junction sites having a functionality $\phi \geq 3$. Interpretation of a comprehensive array of data confined to the region of small strain, however, has given rise to some uncertainties. ${ }^{1-24}$

There have been disagreements, for example, on the origin of some unexpectedly large values measured for the low-deformation modulus, with part of the problem having to do with the definition of entanglements and their possible contributions. ${ }^{25,26}$ For example, the constrained-junction model includes an excess free energy due to restriction of the junction fluctuations from entanglement constraints, but the chains themselves are handled as phantom chains in the configurational space of the network. ${ }^{8,37,38}$ Similarly, in an extension of this model, Erman and Monnerie located the constraints on the centers of mass of the chains rather than only on the junctions. $^{27,28}$

On the other hand, it is universally accepted that on intermediate time scales and at small strains, chain-chain interactions do play an important role. Accordingly, in the single-chain approach, a chain is subjected to a spatial domain of constraints along the entire contour, thus yielding an additional contribution to the elastic free

\footnotetext{
$\dagger$ Permanent address: Department of Chemistry, United Arab Emirates
} University, Box 17551, Al Ain, United Arab Emirates. energy. ${ }^{25,26,29,30}$ In this regard, the recent molecular dynamics simulations of Duering et al. ${ }^{31}$ demonstrated that trapped entanglements contribute to the small-strain modulus of polymer networks. However, the contributions from conserved topologies (trapped entanglements) to the elastic modulus were found to be dependent on the interaction potential employed. ${ }^{31}$

Particularly relevant here are the work of Gottlieb and Gaylord ${ }^{32}$ and the more recent work of Erman and Vilgis ${ }^{33}$ showing that the stress-strain response of a real network is not sensitive to the exact way in which topological constraints are imposed (whether by constraints on the junction fluctuations or by chain-chain interactions). There are significant similarities in the definitions of the associated constrain parameters, in spite of the fact that the proportionality coefficients in these expressions are substantially different. In any case, it is very encouraging that current trends are to reconcile these different points of view, with attempts at increasing rigor.

In this context, it should be noted that the networks that have been most extensively investigated with regard to relating the elastic modulus to network structure have been prepared from PDMS. This particular polymer, unfortunately, has a relatively small value of the plateau modulus, and thus may not be well suited for gauging the influence of topological entanglements latent in the polymer prior to its cross-linking. More relevant polymers exhibiting significantly larger values of the plateau modulus, e.g., poly(butadiene), may be more important in this regard. ${ }^{34}$

Another advance on the experimental side is the better understanding which has been achieved of the hydrosilylation cure often used in the preparation of model 
PDMS networks. Most notably, Macosko and Saam ${ }^{14}$ used vinyl-terminated polyisobutylene to explore this curing approach, even though these reactions were previously considered straight-forward and almost free of side effects. They observed, however, two major side reactions. The first consumes $\mathrm{Si}-\mathrm{H}$ groups to give redistributed siloxane groups in the resulting polymer, as well as gaseous silanes and siloxanes as by-products. The other side reaction, on the other hand, resulted in loss of reactivity of the vinyl groups owing to their shift to an internal position along the chain. These side reactions were reported to be substantial when the experiments were carried out at high temperatures. ${ }^{14,35}$ The maximum value of the modulus occurred at a value of the composition ratio $r \approx 1.2-1.3$, where $r$ is the initial molar ratio of $\mathrm{Si}-\mathrm{H}$ groups to vinyl groups. ${ }^{12-14,35}$ This maximum in the values of the modulus has been observed for both trifunctional and tetrafunctional PDMS networks. It was properly accounted for when the side reactions were considered. ${ }^{10,11}$

In the absence of significant side reactions, balanced stoichiometry $(r=1)$ should lead to a network having the most nearly complete extent of endlinking, and thus have the highest possible value of the modulus. Computer simulation experiments provide further support to this assumption. ${ }^{36}$ Consequently, reported values of the extent of reaction thus obtained are suspect.

The present study focuses on the synthesis of PDMS networks having low degrees of cross-linking, using hydrosilylation reactions at low temperature. Under these conditions, significant side reactions should be absent, and the networks thus formed should be more suitable for comparing molecular theories. ${ }^{10,35}$ The present investigation employs the theory of local constraints on junctions ${ }^{37,38}$ to interpret the elastomeric properties of the networks. Factors affecting the determination of the network parameters will be emphasized with regard to critical examination of the molecular theories of rubberlike elasticity.

\section{ELASTICITY EQUATIONS}

The quantity most often used to analyze results of measurements in uniaxial deformation is the reduced stress or modulus $\left[\mathrm{f}^{*}\right]$ defined by ${ }^{1}$

$$
\left[\mathrm{f}^{*}\right]=f v_{2}{ }^{1 / 3} / A^{*}\left(\alpha-\alpha^{-2}\right)
$$

where $f$ is the equilibrium retractive force, $v_{2}$ the volume fraction of polymer in the network, $A^{*}$ the undeformed cross-sectional area of the sample, and $\alpha=l / l_{0}$ its elongation (ratio of length in the stretched state to the length in the unstretched state at the same volume). Experimental values of $\left[\mathrm{f}^{*}\right]$ for moderate values of elongation can be represented by the simple Mooney-Rivlin phenomenological equation ${ }^{39}$

$$
\left[\mathrm{f}^{*}\right]=2 C_{1}+2 C_{2} \alpha^{-1}
$$

In the affine limit of deformation, the network elastic modulus is given by ${ }^{1}$

$$
\left[\mathrm{f}^{*}\right]_{\mathrm{aff}}=v R T v_{2 \mathrm{C}}^{2 / 3}
$$

where $v$ is the number density of elastically-effective network chains, $R$ the gas constant, $T$ the absolute tempe- rature, and $v_{2 C}$ the volume fraction of polymer chains in the system being crosslinked which were successfully incorporated in the network structure.

The shear (small-strain) modulus $G$ is then expressed by the relationship ${ }^{1,6,12}$

$$
G=\lim _{\alpha \rightarrow 1}\left[\mathrm{f}^{*}\right] \approx 2 C_{1}+2 C_{2}
$$

It is well known that values of $2 C_{1}+2 C_{2}$ tend to overestimate $G$ by about $5 \%$. $1,6,12$

According to the theory of phantom networks, the reduced modulus is 37,38

$$
\left[\mathrm{f}^{*}\right]_{\mathrm{ph}}=\xi R T v_{2 \mathrm{C}}^{2 / 3}
$$

where $\xi$ is the cycle rank of the network related to the network chemical degree of interlinking (i.e., number density of chains). ${ }^{41,42}$ For any type of network, it is given by ${ }^{41,42}$

$$
\xi=v_{\mathrm{a}}-\mu_{\mathrm{a}}=v-\mu
$$

where $v_{\mathrm{a}}$ and $\mu_{\mathrm{a}}$ are the number densities of the elastically-active chains and the elastically-active junctions, respectively, and $\mu$ is the number density of elasticallyeffective junctions.

For a perfect network with its chains joined together by junctions of functionality $\phi$, the cycle rank is given by $1,6,41$

$$
\xi=\left(1-\frac{2}{\phi}\right) v_{\mathrm{a}}
$$

In a perfect network, the number of active junctions $\mu_{\mathrm{a}}$ is $1,6,41$

$$
\mu_{\mathrm{a}}=(2 / \phi) v_{\mathrm{a}}=[2 /(\phi-2)] \xi
$$

Therefore, values of the phantom modulus are based only on contributions from active chemical crosslinks.

The modulus in the phantom limit is related to the phenomenological parameter $2 C_{1}$ by ${ }^{37}$

$$
\left[\mathrm{f}^{*}\right]_{\mathrm{ph}}=\xi R T v_{2 \mathrm{C}}^{2 / 3} \approx 2 C_{1}
$$

Experimental stress-strain measurements give a clear indication that real networks exhibit properties between the phantom and affine limits. ${ }^{37,38,43}$ According to the recent and most comprehensive theory of network behavior by Flory and Erman, the elastic force is taken to be the sum of two contributions 37,38

$$
f=f_{\mathrm{ph}}+f_{\mathrm{c}}
$$

where $f_{\mathrm{ph}}$ is the force predicted from a phantom network and $f_{\mathrm{c}}$ is the contribution to the force arising from local intermolecular entanglements and steric constraints on junction fluctuations. Hence, the expression for $\left[\mathrm{f}^{*}\right]$ in the constraint theory becomes ${ }^{37,38}$

$$
\left[\mathrm{f}^{*}\right]=\xi R T v_{2 C}^{2 / 3}\left(1+f_{\mathrm{c}} / f_{\mathrm{ph}}\right)
$$

The theory predicts that $f_{\mathrm{c}} / f_{\mathrm{ph}}$ decreases with increasing deformation; hence, the modulus would approach the phantom limit at $\alpha \rightarrow \infty$. The relative contribution from the constraints in the limit $\alpha \rightarrow 1$ for a perfect network is predicted by the theory to be $\mathrm{be}^{37,38}$

$$
f_{\mathrm{c}} / f_{\mathrm{ph}}=2 /(\phi-2) \approx 2 C_{2} / 2 C_{1} \text {. }
$$


In this connection, the phenomenological ratio $2 C_{2} / 2 C_{1}$ has been identified with $f_{\mathrm{c}} / f_{\mathrm{ph}} \cdot{ }^{37}$

As pointed out by Flory, ${ }^{42}$ the identification of $v_{\mathrm{a}}$ with $v$ is proper only for perfect networks; otherwise, it is an approximation that is legitimate for high functionality networks. It is worth noting that $v \neq v_{\mathrm{a}}{ }^{42}$ Flory has shown that the effective number of chains for imperfect networks is expressed rigorously by

$$
\nu=2 \xi
$$

Therefore, it follows that for an imperfect network

$$
\left[\mathrm{f}^{*}\right]_{\mathrm{aff}}=v R T=2 \xi R T=2\left(2 C_{1}\right)
$$

In this process, both $v$ and $\mu$ are always larger than $v_{\mathrm{a}}$ and $\mu_{\mathrm{a}}$, provided that the reaction is incomplete.

\section{EXPERIMENTAL}

Vinyl-terminated bifunctional polymers used in this study were obtained from Petrach Chemicals. Standard fractional precipitation techniques were carried out on several samples having different molecular masses at $25^{\circ} \mathrm{C}$. Methyl ethyl ketone was chosen as the solvent and methanol as the precipitant, and initial polymer concentrations were about $5 \%$. The primary aim for this procedure was to decrease the polydispersity index $M_{w} / M_{n}$ of these bifunctional oligomers and to remove unreactive materials (typically cyclics) usually present in commercial PDMS samples. Commercial samples appear to have $2-5 \%$ impurities of $300-1500$ molecular mass.

The samples thus fractionated were exhaustively dried under vacuum at $70^{\circ} \mathrm{C}$ for three days. Their molecular masses and polydispersity indices were obtained by size exclusion chromatography (SEC) using PDMS stan- dards. The polydispersity index was generally below 1.3 in all cases. A series of polymers ranging in molecular masses from 23500 to $7380 \mathrm{~g} \mathrm{~mol}^{-1}$ were utilized to prepare the networks. Specific values and compositions are given in the first two columns of Table I.

The networks were prepared in bulk by mixing the precursor chains with tetrakis(dimethylsiloxy)silane [Si$\left.\left(\mathrm{OSiMe}_{2} \mathrm{H}\right)_{4}\right]$, which was used as received without further purification. Chloroplatinic acid was used as the catalyst and was present in amounts corresponding to $20-30 \mathrm{ppm}$ of elemental $\mathrm{Pt}$ relative to the total weight of the sample. The cross-linking reaction was allowed to proceed in a desiccator for about 5 days at room temperature. The networks thus prepared were removed and turned over and then allowed to cure further for two additional days.

The crosslinked sheets were extracted in toluene for one week to remove any unreacted material; the solvent was changed once after the first two days. The networks, after such extraction, were slowly deswollen in a series of toluene-methanol mixtures of increasing methanol content, and then dried under vacuum. The amounts of extracted material $\omega_{\mathrm{s}}$ were less than $2.5 \%$ for the majority of the networks. Values of the related quantity, $v_{2 c}$, the volume fraction of the polymer successfully incorporated in the network structure, are given in the fourth column of Table I.

Stress-strain isotherms in elongation were obtained on strips cut from the various network sheets. The central test portions of the strips had lengths of about $4.5 \mathrm{~cm}$, widths of about $0.4 \mathrm{~cm}$, and thicknesses of $0.7-1.2 \mathrm{~mm}$. The elongation $\alpha$ was measured using four fiducial marks, nearly $0.75 \mathrm{~cm}$ apart. Stress-strain data were obtained using a sequence of increasing values of $\alpha$.

\begin{tabular}{|c|c|c|c|c|c|c|c|c|c|c|c|c|c|c|c|c|c|}
\hline \multirow{2}{*}{$\frac{M_{n}^{\mathrm{a}}}{\mathrm{g} \mathrm{mol}^{-1}}$} & \multirow{2}{*}{$r$} & \multirow{2}{*}{$\omega_{\mathrm{s}}$} & \multirow{2}{*}{$v_{2 \mathrm{~s}}^{\mathrm{b}}$} & \multirow{2}{*}{$P_{\mathrm{SiH}}$} & \multirow{2}{*}{$P_{\mathrm{vi}}$} & \multirow{2}{*}{$\phi_{\mathrm{e}}$} & \multirow{2}{*}{$v_{2}{ }^{c}$} & \multirow{2}{*}{$\frac{v_{\mathrm{o}} R T^{\mathrm{d}}}{\mathrm{N} \mathrm{mm}^{-2}}$} & \multirow{2}{*}{$\frac{v R T}{\mathrm{~N} \mathrm{~mm}^{-2}}$} & \multirow{2}{*}{$\frac{G}{\mathrm{~N} \mathrm{~mm}^{-2}}$} & \multirow{2}{*}{$\frac{\left[\mathrm{f}^{*}\right]_{\mathrm{ph}}}{\mathrm{N} \mathrm{mm}^{-2}}$} & \multirow{2}{*}{$\frac{2 C_{1}}{\mathrm{~N} \mathrm{~mm}^{-2}}$} & \multirow{2}{*}{$\frac{2 C_{2}}{\mathrm{~N} \mathrm{~mm}^{-2}}$} & \multirow{2}{*}{$\frac{2 C_{2}}{2 C_{1}}$} & \multirow{2}{*}{$\frac{G}{v R T}$} & \multirow{2}{*}{$\frac{2 C_{1}}{\left[\mathrm{f}^{*}\right]_{\mathrm{ph}}}$} & \multirow{2}{*}{$T_{\mathrm{e}}$} \\
\hline & & & & & & & & & & & & & & & & & \\
\hline \multirow[t]{5}{*}{23500} & 0.850 & 0.0441 & 0.163 & 1.000 & 0.773 & 3.45 & 0.640 & \multirow[t]{5}{*}{0.100} & 0.063 & 0.037 & 0.026 & 0.019 & 0.018 & 0.98 & 0.59 & 0.71 & 0.338 \\
\hline & 0.977 & 0.0374 & 0.174 & 0.923 & 0.820 & 3.42 & 0.689 & & 0.068 & 0.129 & 0.028 & 0.047 & 0.082 & 1.74 & 1.91 & 1.67 & 0.422 \\
\hline & 1.097 & 0.0140 & 0.232 & 0.892 & 0.890 & 3.48 & 0.792 & & 0.082 & 0.124 & 0.035 & 0.041 & 0.083 & 2.02 & 1.51 & 1.17 & 0.603 \\
\hline & 1.190 & 0.0858 & 0.130 & 0.735 & 0.795 & 3.21 & 0.589 & & 0.037 & 0.076 & 0.014 & 0.027 & 0.050 & 1.87 & 2.07 & 1.91 & 0.249 \\
\hline & 1.26 & 0.1270 & 0.098 & 0.682 & 0.781 & 3.16 & 0.550 & & 0.025 & 0.055 & 0.009 & 0.016 & 0.039 & 2.42 & 2.17 & 1.71 & 0.171 \\
\hline \multirow[t]{3}{*}{15900} & 1.077 & 0.0704 & 0.142 & 0.805 & 0.788 & 3.27 & 0.614 & \multirow[t]{3}{*}{0.149} & 0.067 & 0.069 & 0.068 & 0.044 & 0.024 & 0.545 & 1.02 & 1.70 & 0.290 \\
\hline & 1.028 & 0.0597 & 0.150 & 0.847 & 0.792 & 3.31 & 0.633 & & 0.077 & 0.144 & 0.069 & 0.078 & 0.066 & 0.849 & 1.88 & 2.57 & 0.324 \\
\hline & 1.216 & 0.1390 & 0.085 & 0.692 & 0.766 & 3.16 & 0.538 & & 0.035 & 0.035 & 0.034 & 0.014 & 0.021 & 1.479 & 1.00 & 1.10 & 0.154 \\
\hline \multirow[t]{5}{*}{10800} & 0.836 & 0.0239 & 0.217 & 1.000 & 0.836 & 3.55 & 0.724 & \multirow[t]{5}{*}{0.219} & 0.154 & 0.151 & 0.049 & 0.070 & 0.081 & 1.149 & 0.98 & 1.04 & 0.479 \\
\hline & 0.923 & 0.0184 & 0.218 & 0.942 & 0.869 & 3.52 & 0.767 & & 0.161 & 0.161 & 0.024 & 0.079 & 0.082 & 1.029 & 1.00 & 1.14 & 0.556 \\
\hline & 1.036 & 0.0687 & 0.158 & 0.773 & 0.801 & 3.25 & 0.618 & & 0.087 & 0.150 & 0.111 & 0.071 & 0.080 & 1.130 & 1.72 & 2.10 & 0.295 \\
\hline & 1.060 & 0.0317 & 0.189 & 0.806 & 0.854 & 3.33 & 0.711 & & 0.124 & 0.228 & 0.120 & 0.108 & 0.121 & 1.122 & 1.85 & 2.18 & 0.454 \\
\hline & 1.200 & 0.0898 & 0.125 & 0.681 & 0.817 & 3.19 & 0.587 & & 0.065 & 0.109 & 0.113 & 0.046 & 0.063 & 1.365 & 1.68 & 1.91 & 0.240 \\
\hline \multirow[t]{5}{*}{7500} & 0.990 & 0.0140 & 0.254 & 0.898 & 0.889 & 3.49 & 0.794 & \multirow[t]{5}{*}{0.315} & 0.234 & 0.246 & & 0.117 & & 1.104 & 1.05 & 1.17 & 0.601 \\
\hline & 1.004 & 0.0124 & 0.253 & 0.892 & 0.896 & 3.49 & 0.805 & & 0.237 & 0.253 & 0.026 & 0.118 & 0.135 & 1.145 & 1.07 & 1.17 & 0.621 \\
\hline & 1.090 & 0.0189 & 0.234 & 0.812 & 0.886 & 3.37 & 0.767 & & 0.203 & 0.187 & 0.026 & 0.107 & 0.081 & 0.757 & 0.92 & 1.29 & 0.551 \\
\hline & 1.226 & 0.0433 & 0.189 & 0.706 & 0.866 & 3.24 & 0.679 & & 0.140 & 0.145 & 0.030 & 0.049 & 0.096 & 1.959 & 1.03 & 0.91 & 0.392 \\
\hline & 1.420 & 0.1072 & 0.133 & 0.601 & 0.853 & 3.15 & 0.573 & & 0.073 & 0.075 & 0.013 & 0.036 & 0.039 & 1.083 & 1.03 & 1.36 & 0.206 \\
\hline \multirow[t]{5}{*}{7380} & 0.867 & 0.0106 & 0.256 & 1.000 & 0.867 & 3.62 & 0.771 & \multirow[t]{5}{*}{0.320} & 0.247 & 0.220 & 0.112 & 0.117 & 0.103 & 0.876 & 0.89 & 1.06 & 0.560 \\
\hline & 0.947 & 0.0084 & 0.270 & 0.960 & 0.909 & 3.63 & 0.835 & & 0.266 & 0.217 & 0.093 & 0.112 & 0.105 & 0.938 & 0.82 & 0.94 & 0.678 \\
\hline & 0.987 & 0.0090 & 0.274 & 0.920 & 0.909 & 3.55 & 0.831 & & 0.257 & 0.223 & 0.100 & 0.118 & 0.106 & 0.901 & 0.87 & 1.04 & 0.668 \\
\hline & 1.015 & 0.0078 & 0.273 & 0.902 & 0.916 & 3.54 & 0.841 & & 0.258 & 0.218 & 0.101 & 0.126 & 0.092 & 0.734 & 0.85 & 1.12 & 0.688 \\
\hline & 1.142 & 0.0106 & 0.256 & 0.803 & 0.917 & 3.39 & 0.819 & & 0.226 & 0.187 & 0.083 & 0.111 & 0.076 & 0.687 & 0.83 & 1.19 & 0.646 \\
\hline
\end{tabular}

Table I. Elastomeric properties of vinyl-terminated poly(dimethylsiloxane) networks prepared at $25^{\circ} \mathrm{C}$

${ }^{a}$ Molecular mass of vinyl-terminated oligomer. ${ }^{\mathrm{b}}$ Volume fraction of polymer at equilibrium swelling in benzene at $25^{\circ} \mathrm{C}$. ${ }^{\mathrm{c}} \mathrm{Volume}$ fraction of elastically effective chains determined by branching theory. ${ }^{15}$ d Number density of elastically effective chains multiplied by $R T$, on the assumption of perfect end-linking. 
Some measurements were taken out of sequence to test for reversibility.

\section{RESULTS AND DISCUSSION}

For networks having $M_{n}=7500$ and $7380 \mathrm{~g} \mathrm{~mol}^{-1}$, the highest possible moduli of elasticity and the lowest values of sol fraction for most of the networks were obtained at a stoichiometric balance (ratio $r=1$ ). Values of the shear (small-strain) modulus $G$ and the sol fraction $\omega_{\mathrm{s}}$ for such networks are presented in Figure 1. Occurence of the maxima in the close viccinity of $r=1$ could serve as a clear indication of the absence of any significant side reactions in the hydrosilylation cure at the lower temperatures employed in this investigation. One should note that the two sets of networks having $M_{n}=7500$ and $7380 \mathrm{~g} \mathrm{~mol}^{-1}$ could be treated as a single set owing to the fact that the difference in molecular masses is far below the allowed experimental errors in the GPC measurements of about $5 \%$.

In the case of networks having $M_{n}=23500 \mathrm{~g} \mathrm{~mol}^{-1}$, however, it is obvious from a closer examination of Figure 2 that values of the modulus go through a judicious maximum at $r \approx 1.06$. This small discrepancy with the value $r=1$ expected in the absence of significant side reactions could be due to inaccuracies in defining the initial stoichiometry.

Occurrence of side reactions results in loss of $\mathrm{Si}-\mathrm{H}$

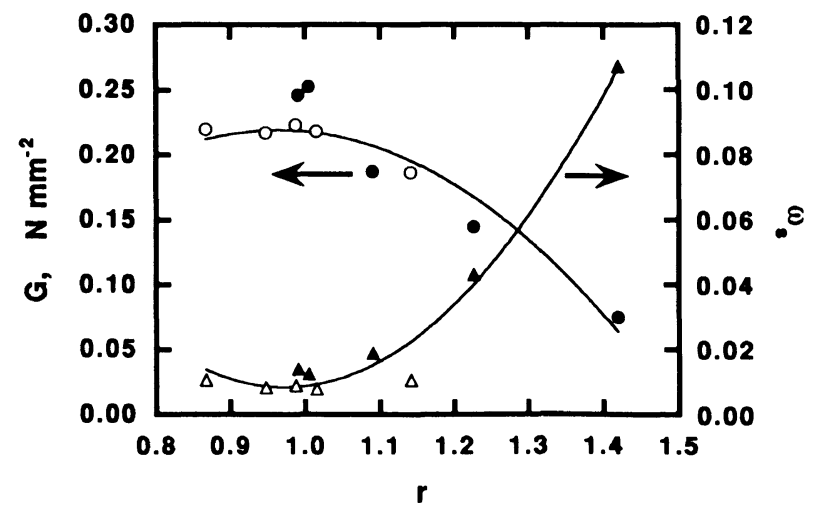

Figure 1. Dependence of the shear modulus and the sol fraction on the stoichiometric imbalance ratios $r$. The open and filled circles represent results obtained for networks having number average molecular masses $M_{n}=7380$ and $7500 \mathrm{~g} \mathrm{~mol}^{-1}$, respectively. The solid lines were located by a second-order polynomial fit for the entire set of data.

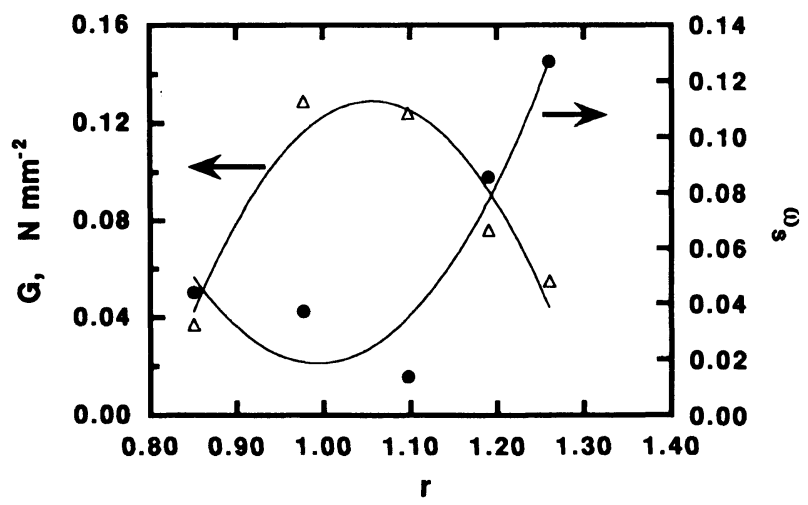

Figure 2. Dependence of the shear modulus and the sol fraction on the stoichiometric imbalance ratios $r$ for networks having number average molecular mass $M_{n}=23500 \mathrm{~g} \mathrm{~mol}^{-1}$. The solid lines were located by a second-order polynomial fit. groups without forming crosslinks, causing the value of $r$ to change. ${ }^{14}$ The observed effective conversions in hydrosilylation cures of vinyl-terminated PDMS oligomers were derived in the same manner used by Macosko and Saam. ${ }^{14}$ Effective conversions to form crosslinks yield the relationships

$$
\begin{gathered}
P_{\mathrm{SiH}}^{\prime}=\frac{P_{\mathrm{SiH}}-P_{\mathrm{Y}}}{1-P_{\mathrm{Y}}} \\
r^{\prime}=r\left(1-P_{\mathrm{Y}}\right)=\frac{P_{\mathrm{vi}}}{P_{\mathrm{SiH}}^{\prime}}
\end{gathered}
$$

where $P_{\mathrm{SiH}}^{\prime}$ is the effective conversion of the $\mathrm{SiH}$ groups to crosslinks, $P_{\mathrm{Y}}$ the extent of loss of $\mathrm{SiH}$ groups through volatilization, and $r^{\prime}$ the effective value of the stoichiometric ratio. In any case, the corrected values of $r^{\prime}$ $\left(r / r^{\prime} \approx 1.06\right)$ have been used to obtain the structure parameters for the networks having $M_{n}=23500 \mathrm{~g} \mathrm{~mol}^{-1}$. Also, it is worth noting here the lower values of $v_{\mathrm{a}}$ and $\mu_{\mathrm{a}}$, computed from branching theory, ${ }^{15}$ as compared with those of $v_{0}$ that correspond to perfect end-linking.

In the present analysis, the network structural parameters $v_{\mathrm{a}}, \mu_{\mathrm{a}}$, as well as the effective functionality $\left(\phi_{\mathrm{e}}=\right.$ $\left.2 v_{\mathrm{a}} / \mu_{\mathrm{a}}\right)$, the volume fraction of the elastically effective chains $v_{2}$, and the fraction of trapped entanglements $T_{\mathrm{e}}$ were calculated from the sol fraction $\omega_{\mathrm{s}}$ using the branching theory. ${ }^{15}$ In order to account for the side reactions in the hydrosilylation cures mentioned above, the corrected values $r^{\prime}$ were used instead of $r$ in the pertinent calculations, for networks having $M_{n}=23500 \mathrm{~g} \mathrm{~mol}^{-1}$. Values of the extent of reaction $P_{\mathrm{SiH}}$, and subsequently different structural parameters of the network, were obtained by an iterative solution of eq A5 through A7 in ref 15 . Calculated values of the affine modulus $\left[\mathrm{f}^{*}\right]_{\mathrm{aff}}$ and the phantom modulus $\left[\mathrm{f}^{*}\right]_{\mathrm{ph}}$ were obtained from eq 3 and 5, respectively. The results thus obtained are presented in Table I.

In this connection, one should note that it is difficult to obtain accurate values of the sol fraction particularly when the quantities measured are small. Nontheless, the branching theory was used to calculate the structure parameters of the network to the extent that it is the best alternative at hand. For the tetrafunctional networks, thus prepared, values of $v_{\mathrm{a}}$ and $\mu_{\mathrm{a}}$, determined by branching theory, were used as an approximate substitute for $v$ and $\mu$, even though they are known to be nonequivalent.

It is important to note here that network imperfections generated by the incompleteness of end-linking would act as diluent, even in the dry unswollen state. ${ }^{44}$ As shown in eq 1 , the real values of both $G$ and $\left[\mathrm{f}^{*}\right]_{\mathrm{ph}}$ should be reduced by $v_{2}^{-1 / 3}$ (where $v_{2}$ is the volume fraction of elastically-effective chains in the network). Hence, values of $\left[\mathrm{f}^{*}\right]_{\mathrm{aff}}$ and $\left[\mathrm{f}^{*}\right]_{\mathrm{ph}}$ predicted by eq 3 and 5 would be

$$
\begin{aligned}
& {\left[\mathrm{f}^{*}\right]_{\mathrm{aff}}=v R T v_{2 C^{2} v_{2}^{-1 / 3}}^{2}} \\
& {\left[\mathrm{f}^{*}\right]_{\mathrm{ph}}=\xi R T v_{2 C^{2} v_{2}^{-1 / 3}}}
\end{aligned}
$$

where the factor $v_{2}^{-1 / 3}$ has not been accounted for in obtaining the reported experimental values of both $G$ and $2 C_{1}$. (In all pertinent calculations values $v_{\mathrm{a}} R T$ thus calculated are corrected by the same factor.) The corrected values are reported in Table I. 


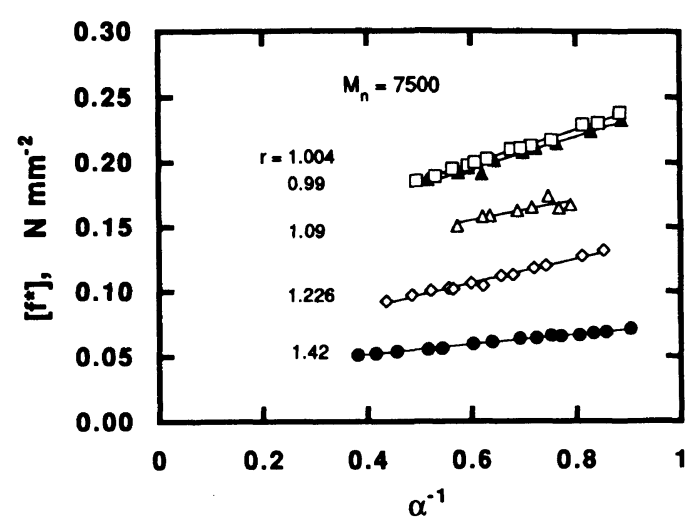

Figure 3. Stress-strain isotherms for the networks having number average molecular mass $M_{n}=7500$ at $25^{\circ} \mathrm{C}$. Each isotherm is labelled with the stoichiometric ratio $r$. The isotherms are shown as suggested by the Mooney-Rivlin analysis, and the straight lines have been located by least-squares analysis.

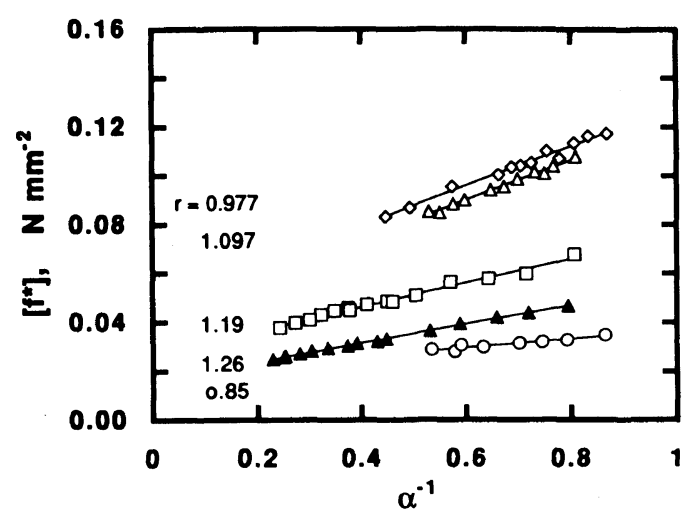

Figure 4. Stress-strain isotherms for the networks having number average molecular mass $M_{n}=23500 \mathrm{~g} \mathrm{~mol}^{-1}$. See legend to Figure 3 .

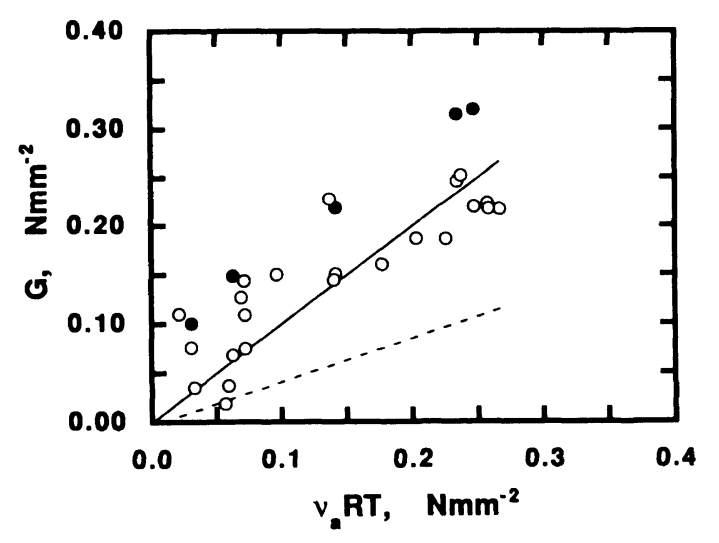

Figure 5. The small-strain modulus $G$ shown as a function of a measure of the degree of the cross-linking of the networks, as obtained from the active chain density $v_{\mathrm{a}}$. The solid line is for the affine modulus itself, eq 17. The experimental data are denoted by open circles, while the filled circles represent results calculated on the assumption of perfect network formation.

The stress-strain data were interpreted in terms of the reduced stress or modulus, as defined in eq 1 . The equilibrium values of $\left[\mathrm{f}^{*}\right]$ were plotted against the reciprocal elongation $\alpha^{-1}$ as suggested by the MooneyRivlin procedure, embodied in eq 2 , and the straight lines through the isotherms were located by least-squares analysis. Representative stress-strain isotherms are illustrated in Figures 3 and 4 . Values of the constants $2 C_{1}$ and $2 C_{2}$ thus obtained are reported in Table $I$.

Figure 5 shows values of $G$ plotted against $v_{\mathrm{a}} R T$. The

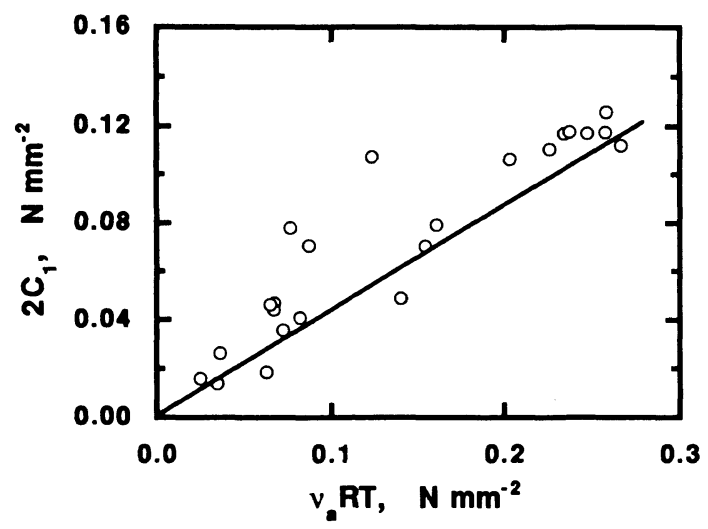

Figure 6. The Mooney-Rivlin estimate of the high-deformation modulus $2 C_{1}$ shown as a function of a measure of the degree of the crosslinking. The solid line represents the phantom limit, according to eq 18 .

solid line represents the upper bound of theory (the affine modulus), eq 18. The dashed line shows values of the phantom modulus $\left[\mathrm{f}^{*}\right]_{\mathrm{ph}}$ calculated according to eq 17 . Although the data scatter somewhat, more or less satisfactory agreement with theory could be observed, within the limits set by experimental error. At the lower degrees of cross-linking, some values of $G$ exceed those predicted by the theory. The data, however do not suggest an appreciable intercept with the origin that could be attributed to contributions from trapped entanglements. Hence, the small-strain modulus $G$ must at least approximately vanish in the limit $v_{\mathrm{a}} R T \rightarrow 0$.

In this connection, the enhancement of [f*] at $\alpha \rightarrow 1$ has been observed to vanish upon swelling, suggesting that in some cases it may be due to difficulties in reaching elastic equilibrium when the network chains are very long. ${ }^{6,8}$ Also, the results manifest a trend for departure of the values of $G$ from the upper bound of the constrained theory as $v_{\mathrm{a}} R T$ increases. Such decreases in values of $G$ with increase in cross-link density are expected because of the decrease in interpenetration as the network chain length decreases. ${ }^{6,11}$

In Figure 6, values of $2 C_{1}$ are shown as a function of $v_{\mathrm{a}} R T$. They were interpreted using eq 4 , and the solid line represents theory according to eq 17 . In other words, it would represent the network connectivity as determined from the phantom network topology, according to eq 5 and 6 . Although the experimental results scatter somewhat, satisfactory agreement between theory and experiment is apparent.

It is of great importance to underline the fact that values of $v_{\mathrm{a}}$ are used as an approximate substitute for those of $v$, even though they are known to be nonequivalent. Nonetheless, the results should be suitable for the present purposes. At this point in the discussion, it is noteworthy that the formation of cyclics during network formation could give a higher value of the sol fraction even if a perfect network is formed. ${ }^{6,36}$ This seems to be true by observing that values of $G$ and so $2 C_{1}$ that exceeded those predicted by theory correspond nearly to values of $v_{0} R T$ calculated on the assumption of the formation of perfect networks, $c f$. Table I and Figure 5. Also, for the networks showing the most divergence, calculated values of $\left[\mathrm{f}^{*}\right]_{\mathrm{ph}}$ are two to threefold lower than the corresponding values of $2 C_{1}$ owing to inherent inaccuracies (discussed previously) in the 


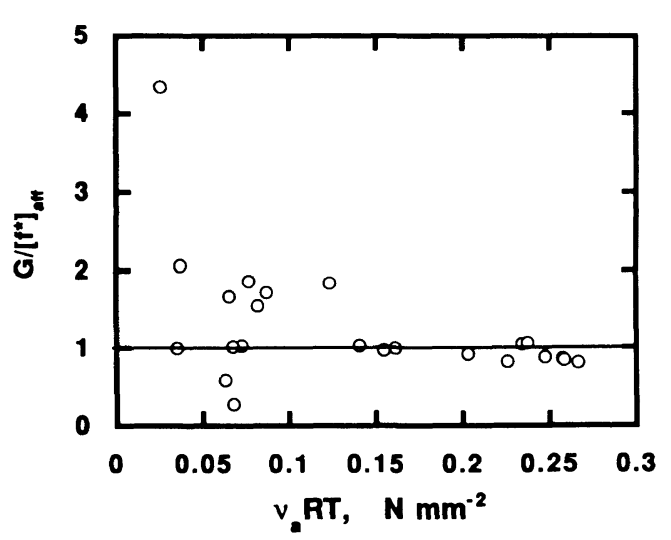

Figure 7. Values of the ratio $G / v_{\mathrm{a}} R T$ of the experimental small-strain modulus to the calculated affine modulus for the trifunctional PDMS networks shown as a function of a measure of the degree of the cross-linking. The solid line is for the affine modulus.

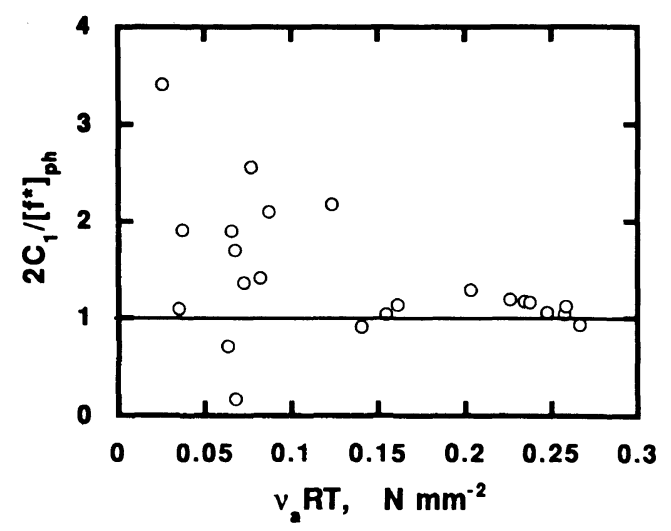

Figure 8. Values of the ratio $2 C_{1} /\left[\mathrm{f}^{*}\right]_{\mathrm{ph}}$ of the experimental largestrain modulus to the calculated phantom modulus for the trifunctional PDMS networks shown as a function of a measure of the degree of the cross-linking. The solid line is for the phantom modulus.

determination of the sol fraction. This is contrary to experimental and theoretical assumptions where $\left[\mathrm{f}^{*}\right]_{\mathrm{ph}}$, expressed by eq 9 , holds for any network regardless of its functionality or the presence of defects (such as chains connected to the network at only one end). As also mentioned above, a wealth of experimental data identify the phantom modulus $\left[\mathrm{f}^{*}\right]_{\mathrm{ph}}$ with the phenomenological constant $2 C_{1} \cdot{ }^{1-11,37}$ The departure observed at low values of $v_{\mathrm{a}} R T$ has been the major problem in the study of rubberlike elasticity and may simply be due to experimental difficulties. As such, the behavior observed is within the limits of the constrained-junction theory of Flory and Erman. ${ }^{37,38}$

For further elaboration, it is also useful to show the ratios $G /\left[\mathrm{f}^{*}\right]_{\text {aff }}$ and $2 C_{1} /\left[\mathrm{f}^{*}\right]_{\mathrm{ph}}$ of the small-strain and large-strain moduli to the theoretical values of the modulus, respectively. According to eq 3-5, the maximum expected values are unity. The dependence of such values on $v_{\mathrm{a}} R T$ is represented in Figures 7 and 8 , respectively. Again one notes the same divergences discussed above, more specifically, for networks having low degrees of cross-linking. Such values are certainly overestimated and this could be mainly due to difficulties in reaching elastic equilibrium when the network chains are very long or to higher slopes in Mooney-Rivlin plots. ${ }^{39}$ Inaccuracies in determination of the stoichiometric ratios $r$ as well as other difficulties already men-

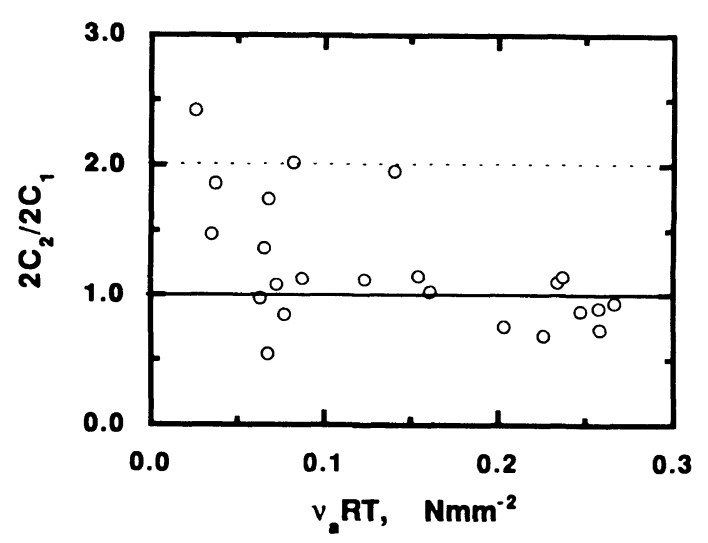

Figure 9. The dependence of the ratio $2 C_{2} / 2 C_{1}$ on a measure of the degree of the cross-linking, shown as a measure of the active chain density $v_{\mathrm{a}}$. The solid line represents the values predicted on the assumption of tetrafunctional networks and the dotted line is that for trifunctional networks, according to eq 12 .

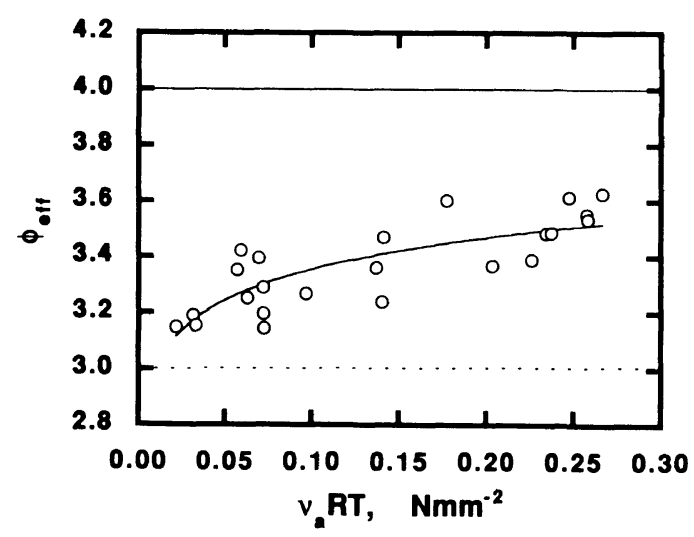

Figure 10. The dependence of the effective functionality of the networks on a measure of the degree of the cross-linking. The solid line represents the values predicted on the assumption of perfect tetrafunctional networks and the dotted line is that for perfect trifunctional networks.

tioned might also lead to such a divergence. Once again, the mere fact that almost all values of $G /\left[\mathrm{f}^{*}\right]_{\text {aff }}$ and $2 C_{1} /\left[\mathrm{f}^{*}\right]_{\mathrm{ph}}$ are equal or less than values of the asymptotes predicted by the theory lends support to suggestions that trapped entanglements play only a minor role in these networks.

The ratio $2 C_{2} / 2 C_{1}$ is predicted to reach a maximum value of unity for tetrafunctional networks, as shown in eq 12. The experimental values are shown in Figure 9. Some data points exhibit lager values than the asymptotic value of unity at low degrees of cross-linking. Such a divergence could be attributed mainly to changes in the effective functionality of the network. The functionality is found to approach a value of 3 for the networks having smaller values of $v_{\mathrm{a}} R T$. The calculated values of $\phi_{\text {eff }}$ are shown in Figure 10. This would mean that the majority of the junctions for such networks are essentially trifunctional. For trifunctional networks, the ratio $2 C_{2} / 2 C_{1}$ is predicted to reach an asymptotic value of 2 , particularly for networks having high values of $M_{n}$ and so with the high degree of chain interpenetration required for affine behavior. This is consistent with the interpretation based on the constrained-junction theory.

In interpretations based on postulating contribution to the modulus from trapped entanglements, the more 


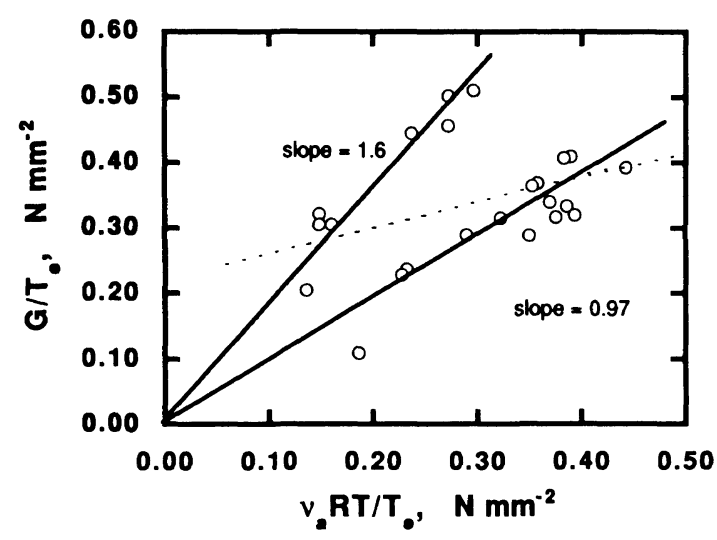

Figure 11. Langley plot of values of the shear (small-strain) modulus reduced by the trapped entanglement factor $T_{\mathrm{e}}$ shown as a function of a measure of the degree of cross-linking reduced by $T_{\mathrm{e}}$, according to eq 20.

general relationship for the modulus in the small-strain limit has been proposed by Dossin and Graessley to be 19,20

$$
G=\left(v_{\mathrm{a}}-h \mu_{\mathrm{a}}\right) R T+G_{\mathrm{e}} T_{\mathrm{e}}
$$

Here $h$ is an empirical parameter between 0 and 1 that allows for real network behavior between the affine and phantom limits, and can be expressed in terms of the parameters $\kappa$ and $\zeta$ in the Flory-Erman theory. ${ }^{37}$ The quantity $G_{\mathrm{e}}$ is the entanglement modulus (frequently approximated by the plateau modulus which is about $0.24 \mathrm{~N} \mathrm{~mm}^{-2}$ for PDMS), and $T_{\mathrm{e}}$ is the fraction of entanglements permanently trapped by the network. ${ }^{19,20}$ Equation 19 can be rewritten as

$$
\frac{G}{T_{\mathrm{e}}}=G_{\mathrm{e}}+\left(1-h \frac{\mu_{\mathrm{a}}}{v_{\mathrm{a}}}\right) \frac{v_{\mathrm{a}} R T}{T_{\mathrm{e}}}
$$

In Figure 11, the shear modulus data are shown as suggested by eq 20 . The interesting point here is that the data points are seen to be well represented by two straight lines intersecting the origin, within limits set by the scattering of data points. As already discussed, this behavior can be attributed to difficulties encoutered in obtaining accurate values of the sol fraction and thus the various structural parameters of the network derived from it. It is necessary to comment on the upper set of data points. The observed slope would give rise to a negative value of $h(c f . \approx-1.2)$ which, if real, would be at variance with theory and the physical meaning of the different terms of eq 20. It is to be noted here that Queslel and Mark observed different sets of data lines in similar plots having different values of $h$, in accord with the present observations. ${ }^{6,36}$ The dotted line represents all data points as treated by least-squares analysis. An intercept of $\approx 0.22$ and $h \approx 1.0$ (i.e., no suppression of fluctuations ${ }^{12}$ ) is obtained. However, a poor correlation coefficient of 0.4 is gotten.

Excluded in Figure 12 are the data points corresponding to networks with high sol fraction (greater than 0.06) as well as those that are presumed to be perfectly endlinked, as noted before, even though overestimated values of the sol fraction were obtained. ${ }^{6,36}$ This procedure could be valid in view of the negative value of $h$ observed for these data points. The least-squares line is seen to intersect the origin, within limits set by the scattering of

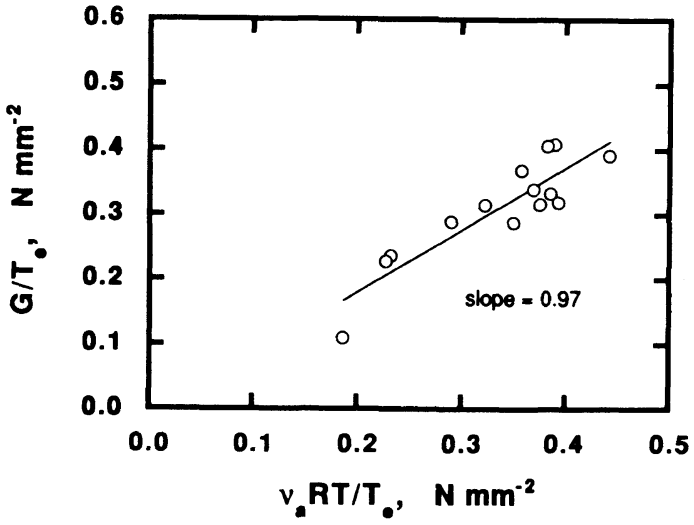

Figure 12. The comparisons made in Figure 11 but now for networks with low sol fraction, less than 0.06 .

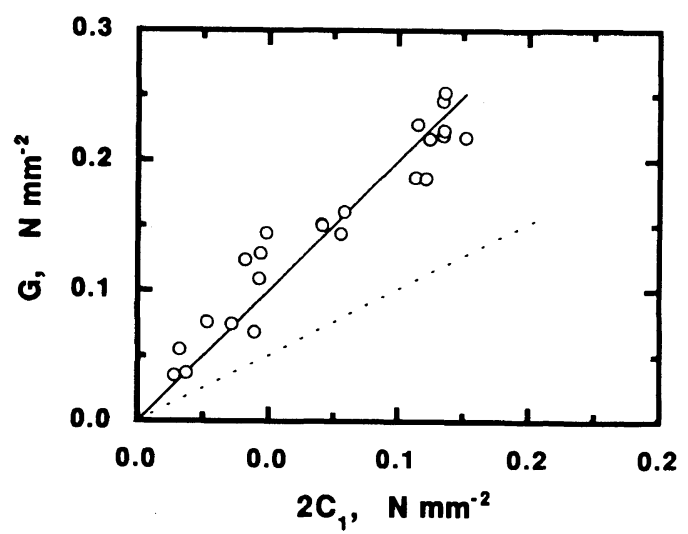

Figure 13. The modulus shown as a function of the Mooney-Rivlin estimate of the high-deformation modulus $2 C_{1}$. The solid line is for the affine limit for an imperfect network as approximated by $2 \times\left(2 C_{1}\right)$, calculated according to eq 14 . The dotted line is for the phantom modulus approximated by $2 C_{1}$.

data points. The slope is 0.97 , as would be expected for total suppression of fluctuations. An excellent correlation coefficient of 0.9 is obtained. As such, it is apparent that $G_{\mathrm{e}} \approx 0$, and consequently the small-strain modulus should vanish likewise in the limit $\mu_{\mathrm{e}} / T_{\mathrm{e}} \rightarrow 0$. Assigning a constant value of $h$ to networks having different degrees of cross-linking is contrary to rigorous theory where the suppression of fluctuations is dependent on the degree of cross-linking. ${ }^{6,45}$ In any case, the results obtained do not provide evidence for significant contributions to the small-strain modulus from trapped entanglements.

In view of the serious difficulties discussed above, the networks prepared as described are admittedly imperfect. Also, a last argument could be the difficulties arising from inhomogeneities in the cross-linking process, as has been pointed out elsewhere. ${ }^{46}$ A straight-forward method to test the validity of the predictions of the theory of Flory and Erman consists of plotting $G \approx$ $2 C_{1}+2 C_{2}$ against $2 C_{1} \approx\left[\mathrm{f}^{*}\right]_{\mathrm{ph}}$ in order to overcome the cited difficulties in obtaining accurate values of the network structural parameters. As previously pointed out, $2 C_{1}$ is proportional to the effective interconnectivity of the network, and therefore can be used to define an effective number of chains $v$ and junctions $\mu$, regardless of the incompleteness of the network formation process. $^{9,37}$ The data thus obtained are plotted in Figure 13. In this figure, the dotted line represents the lower bound of the theory, the phantom limit, and the solid 
line approximates the upper bound, the affinely-deforming network, calculated according to eq 17 . The results are well represented within the upper bound of the theory. This procedure circumvents complications in accurately determining the network parameters. As is readily seen, the results are in accord with the main premises of the constrained-junction theory and the universal treatment of imperfect networks set forward by Flory. ${ }^{42}$

Acknowledgments. It is a pleasure to acknowledge the financial support provided JEM by the National Science Foundation through Grant DMR-9422223 (Polymers Program, Division of Materials Research). Also, we express our thanks to Ms. M. Mostafa for help in the preparation of some networks.

\section{REFERENCES}

1. J. E. Mark and B. Erman, "Rubberlike Elasticity. A Molecular Primer," Wiley-Interscience, New York, N.Y., 1988.

2. J. E. Mark and J. L. Sullivan, J. Chem. Phys., 66, 1006 (1977).

3. J. E. Mark, R. R. Rahalkar, and J. L. Sullivan, J. Chem. Phys., 70, 1749 (1979).

4. M. A. Llorente and J. E. Mark, J. Chem. Phys., 71, 682 (1979).

5. M. A. Llorente and J. E. Mark, Macromolecules, 13, 681 (1980).

6. J. P. Queslel and J. E. Mark, Advances in Polym. Sci., 17, 229 (1985).

7. B. Erman, W. Wagner, and P. J. Flory, Macromolecules, 13, 1554 (1980).

8. B. Erman and P. J. Flory, Macromolecules, 15, 806 (1982).

9. P. J. Flory and B. Erman, J. Polym. Sci., Polym. Phys. Ed., 22, 49 (1984).

10. M. A. Sharaf, A. S. Alshamsi, and J. E. Mark, Preprints, Polym. Mater. Sci. Eng., 68, 319 (1993).

11. M. A. Sharaf and J. E. Mark, Polymer, 35, 740 (1994).

12. M. Gottlieb, C. W. Macosko, G. S. Benjamin, K. O. Meyers, and E. W. Merrill, Macromolecules, 14, 1039 (1981).

13. C. W. Macosko and G. S. Benjamin, Pure Appl. Chem., 53, 1505 (1981)

14. C. W. Macosko and J. C. Saam, Polym. Bulletin, 18, 463 (1987)

15. E. M. Valles and C. W. Macosko, Macromolecules, 12, 673 (1979).

16. M. Gottlieb, C. W. Macosko, and T. P. Lepsch, J. Polym. Sci.,
Polym. Phys. Ed., 19, 1603 (1981).

17. S. K. Patel, S. Malone, C. Cohen, J. R. Gilmore, and R. H. Colby, Macromolecules, 25, 5241 (1992).

18. N. P. Langley, Macromolecules, 1, 348 (1968).

19. L. M. Dossin and W. W. Graessley, Macromolecules, 12, 123 (1979).

20. D. S. Pearson and W. W. Graessley, Macromolecules, 13, $1001(1980)$

21. K. O. Meyers, M. I. Bye, and E. W. Merrill, Macromolecules, 13, $1045(1980)$.

22. K. A. Kirk, S. S. Bidstrup, E. W. Merrill, and K. O. Meyers, Macromolecules, 15, 112 (1982)

23. W. Oppermann and N. Rennar, Prog. Coll. Polym. Sci., 75, 49 (1987).

24. W. Oppermann and N. Rennar, Colloid. Polym. Sci., 270, 527 (1992).

25. G. Heinrich, E. Straub, and G. Helmis, Adv. Polym. Sci., 85, 33 (1988), and references cited therein.

26. N. R. Langley, J. Noncryst. Solids, 131-133, 894 (1991), and references cited therein.

27. B. Erman and L. Monnerie, Macromolecules, 22, 3342 (1989).

28. B. Erman and L. Monnerie, Macromolecules, 25, 4456 (1992).

29. R. C. Ball, M. Doi, S. F. Edwards, and M. Warner, Polymer, 22 , 1010 (1981)

30. S. F. Edwards and T. A. Vilgis, Rep. Prog. Phys., 51, 243 (1988).

31. E. R. Duering, K. Kremer, and G. S. Grest, Macromolecules, 26, 3241 (1993).

32. M. Gottlieb and R. Gaylord, Polymer, 24, 1644 (1983).

33. B. Erman and T. A. Vilgis, Macromolecules, 26, 6657 (1993).

34. M. I. Aranguren and C. W. Macosko, Macromolecules, 21, 2484 (1988).

35. K. V. Sundar, L. Coyne, F. Chambon, M. Gottlieb, and H. M. Winter, Polymer, 30, 2222 (1989).

36. Y. Leung and B. E. Eichinger, J. Chem. Phys., 80, 3885 (1984).

37. P. J. Flory, J. Chem. Phys., 66, 5720 (1977).

38. P. J. Flory and B. Erman, Macromolecules, 15, 800 (1982).

39. M. Mooney, J. Appl. Phys., 1, 582 (1940); R. S. Rivilin, Philos. Trans. Roy. Soc. London, Ser. A, 241, 379 (1948).

40. H. M. James and E. Guth, J. Chem Phys., 15, 669 (1947).

41. G. Ronca and G. Allegra, J. Chem. Phys., 63, 4990 (1975).

42. W. W. Graessley, Macromolecules, 8, 186 (1975); D. S. Pearson and W. W. Graessley, Macromolecules, 11, 528 (1978).

43. P. J. Flory, Macromolecules, 15, 99 (1982).

44. B. Erman and J. E. Mark, Macromolecules, 20, 11 (1987).

45. B. Erman and P. J. Flory, Macromolecules, 15, 806 (1982).

46. J. R. Falender, G. S. Y. Yeh, and J. E. Mark, Macromolecules, 12, 1207 (1979) 